\title{
Facilitation of completely paralyzed forearm muscle activity in chronic stroke patients
}

\author{
García-Cossio E., Member, IEEE, Birbaumer N. and Ramos-Murguialday A., Member, IEEE
}

\begin{abstract}
Stroke is the main cause of hemiparesis in developed countries. Very often upper limbs are compromised and the hemiparesis is characterized by abnormal muscle activations especially at the level of the wrist and fingers (distal muscles). In this study we investigated the stability and strength of paretic upper limb muscle activity during different bilateral movements eliciting different postures and muscle recruitment. We recorded surface EMG of 45 severe chronic stroke patients without residual finger extension during six bimanual movements. Sixteen bipolar-EMG electrodes were placed on both upper limbs at the level of proximal and distal areas. We extracted the waveform length from the EMG data, in order to investigate muscle activity level at the paralyzed muscles during all movements. Our results indicated that different positions during multi-joint movements of the upper limb facilitated the contraction of the affected muscles (forearm extensors) involuntarily during the movement in which this activation was not expected (e.g. abduction of the upper arm) in more than $64 \%$ of the patients. Here, we show that severe affected chronic stroke patients can induce a higher activation of the paretic muscles of the forearm by changing the upper limb posture. This might be an important hint to design multijoint coordinated movements involving proximal and distal musculature for stroke motor rehabilitation.
\end{abstract}

\section{INTRODUCTION}

More than $75 \%$ of stroke survivors present upper limbs motor impairments resulting in hemiparesis [1], which is characterized by abnormal muscle activations especially at the level of the wrist and fingers (distal muscles) [2]. If most paralyzed muscles (normally at distal joints) can elicit EMG activity, this is normally unstable and very or extremely weak. Therefore voluntary motion in the severe impairment

*This is work was funded by the Deutsche Forschungsgemeinschaft (DFG Bi195), the Werner Reichardt Center of Integrative Neuroscience (CIN 2011-18), Bernstein 01GQ0761, BMBF 16SV3783, European Union (ERC 227632, HUMOUR 231724, WAY 288551), Baden Würtemberg Stiftung (ROB-1), DGIST, and funded by the Ministry of Education, Science and Technology of Republic of Korea, and DAAD (Deutscher Akademischer Austauschdienst).

García Cossio E. is with the Institute of Medical Psychology and Behavioral Neurobiology and MEG Center, University of Tubingen, Silcher Str. 5, 72070 Tübingen, Germany

Birbaumer N. is with Institute of Medical Psychology and Behavioral Neurobiology and MEG Center, University of Tubingen, Silcher Str. 5, 72070 Tübingen, Germany; and the Ospedale San Camillo, Istituto di Ricovero e Cura a Carattere Scientifico, Via Alberoni, 70, 30126 Venezia Lido, Italy

Ramos-Murguialday A. is with the Institute of Medical Psychology and Behavioral Neurobiology and MEG Center, University of Tubingen, Silcher Str. 5, 72070 Tübingen, Germany; and with TECNALIA, Health Technologies Department, Mikeletegi Pasalekua 1, 20009 San Sebastian, Spain. state is either not feasible or, if the motor behavior can be initiated, is tied by abnormal muscle synergies (i.e muscle activity patterns) [3]. Previous studies have shown that abnormal synergies are caused by abnormal co-activation of muscles being these almost stereotypical (flexor and extensor synergies) [4]. Therefore, patients cannot exert independent joint control during movement. Other studies have shown that after motor rehabilitation these synergies can be break down and the voluntary movements can be facilitated [5].

More than three decades ago, a previous study already underlined the relation between the hemiplegic's postural situation (e.g. sitting, standing) and his ability to perform selective voluntary muscle action [6]. These approaches consider that the basic postural orientation of the individual relative to the base of support and gravity determines the movement strategies that will be accessible and effective [6]. The alignment of body segments at both the initiation of movement and throughout the evolvement of movement plays a critical role in the postural control strategies used and therefore in the muscle activity that can be elicited [7]. For accurate multi-joint movements, the CNS must control for the effects of interaction torques arising from motions at other joints [8]. Appropriate postural control and the ability to move selectively facilitate the production of coordinated sequences of movement referred to as movement patterns [9].

Here we investigated the stability and strength of the EMG activity in the completely paralyzed muscles of the upper limb during the execution of movements involving different positions and therefore different muscle recruitment in 45 severely affected chronic stroke patients. We hypothesize that different upper limb postures and the involvement of less paralyzed and more stable upper arm muscles (during multi-joint movements) influence and facilitate directly EMG control of the most compromised muscles at the level of the forearm.

\section{Materials AND Methods}

\section{A. Patients}

45 chronic stroke patients (mean age 55 and mean time after stroke 72.3 months) were recruited via public information (German stroke associations, rehabilitation centers, hospitals) all over Germany.

Patients were selected according to a strict selection criteria, including: 1) no residual finger extension; 2) time since stroke of at least 10 months; 3) no psychiatric or neurological condition other than stroke (for more details see [10]). On average the patients presented upper limb motor scale Fugl-Meyer Assessment scale (cFMA) scores (excluding items assessing upper extremity sensation and pain, coordination, speed and reflexes, see [10]) of $11.9 \pm 8.8$ 
from a maximum of 54 points. The study was approved by the ethics committee of the Faculty of Medicine of University of Tübingen, Germany.

\section{B. Task and EMG recordings}

The EMG activity was recorded by either a 32-channel unipolar or a 16 bipolar BrainAmp (Brainproducts $\mathrm{GmbH}$, Munich Germany) from the non-paretic and paretic upper limb using sixteen bipolar electrodes (eight on each limb) placed on top of: 1) extensor carpi ulnaris, 2) extensor digitorum, 3) flexor carpi radialis, palmaris longus, flexor carpi ulnaris (flexion), 4) long head of the biceps (flexion), 5) the external head of the triceps, 6) anterior portion of deltoid muscle, 7) lateral portion of deltoid muscle and 8) posterior portion of deltoid over the teres minor and infraspinatus muscles (Fig. 1-A).

Patients were asked to perform six different arm and hand movements: 1) flexion and 2) abduction of the upper arm, 3) extension of the elbow, 4) supination/pronation, 5) wrist extension and 6) finger extension (Fig. 1-C). These movements were selected because of their similarity to movements performed during the upper limb motor scores of the FMA. EMG data during these movements were used to quantify rehabilitation efficacy of BCI in chronic stroke comparing EMG before and after intervention (see [10]).
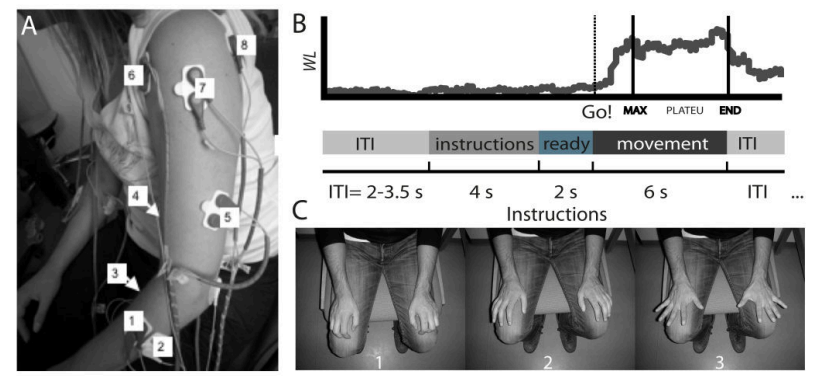

Figure 1. A- Electrode montage. B- Experimental design of the task. Patients were instructed to rest during a randomized period of 2 to $3.5 \mathrm{~s}$ (ITI), after they were presented with some images indicating the task to perfomed (C) the ready interval of $2 \mathrm{~s}$ was showed and after a Go! cue appeared indicating the beginning of the movement. The EMG activity (represented with the waveform length (WL)) is shown in each of the steps of the experiment. After the Go! cue the muscle activity starts increasing until a maximum (max) value. Subsequently, it is stabilized in a plateu until the movement is finished (end) and the EMG activity returns slowly to baseline. (C)-Representation of stages of motion in one of the task: finger extension.

Patients were instructed to perform each movement with both arms simultaneously after a "Go!" cue maintaining the final posture during six seconds (Fig. 1-B and C-3) until a "Rest" cue was presenting indicating them to relax. Compensatory movements were discouraged. The experiment consisted in 3 or 4 blocks where each block was composed by 10 trials for each of the six movements (in total 60 trials per block).

\section{EMG pre-processing}

If EMG data were acquired using the unipolar amplifier data were bipolarized. EMG data were notch filtered at $50 \mathrm{~Hz}$ and band pass filtered between $10 \mathrm{~Hz}$ and $500 \mathrm{~Hz}$. EMG signals were rectified and visually inspected by an expert for artifact rejection (like poor skin contact, amplifier saturation). The data was epoched from - $9 \mathrm{sec}$ to $9 \mathrm{sec}$ relative to the
"Go!" cue and concatenated for each task (Fig. 1-B). In order to avoid drifts in the data, the EMG signals were baseline corrected using as a baseline the average signal from -7 to -2 sec relative to the "Go!" cue. Independent component analysis was applied considering all movements. Eight principal components were used for artifact rejection (e.g. incorrect movement). A $200 \mathrm{msec}$ sliding window with a 180 msec overlap was used to calculate the Waveform Length (WL) of the signal [11], which provides indicators for signal amplitude and frequency. The WL estimates the complexity of the EMG waveform and can be calculated as it is shown in (1).

$$
W L=\sum_{k=1}^{L}\left|x_{k}-x_{k-1}\right|
$$

$\mathrm{L}$ being the length of the window used in the estimation of the waveform length and $X_{k}$ being the amplitude of the filtered and rectified EMG signal at time point $\mathrm{k}$.

\section{Thresholding and comparison of amplitude and stability of the EMG data across movements.}

Two different forearm thresholds were used for calculating the amount of trials presenting higher WL amplitude in forearm muscles during upper arm movements than during forearm movements. The first forearm threshold was calculated by taking the mean plus one standard deviation (mean+std) of the activity across trials of each of the forearm muscles during finger extension (see horizontal continuous lines in Fig. 2) and wrist extension, independently. This threshold represented the mean EMG activity in forearm extensor muscles. In order to guarantee that this difference was statistically significant, the 95percentile across trials of each forearm muscle EMG activity level (i.e. WL amplitude) during finger extension (see horizontal dashed lines in Fig. 2) and wrist extension was used as the second forearm threshold.

We compared the mean activity of each trial during 1) flexion, 2) abduction of the upper arm, 3) extension of the elbow and 4) supination/pronation against each forearm threshold calculated for extension and wrist extension (e.g. Fig. 2 shows that in most of the trials the forearm thresholds during finger extension were exceeded during flexion of upper arm). The percentage of trials exceeding each threshold during each upper arm movement was calculated for each patient. In order to see whether EMG activity during upper arm movements was not only bigger (e.g. Fig. 2) but also more stable and controllable than during forearm movements (e.g. Fig. 2-A), we calculated the percentage of patients that showed in more than $50 \%$ of the upper arm movement trials a WL amplitude higher than each forearm threshold.

\section{RESULTS}

\section{A. Waveform length}

Figure 2 illustrates WL amplitude of forearm muscles calculated for two different patients (P1 and $\mathrm{P} 2)$ during finger extension and flexion of the upper arm in 40 (P1) and 30 (P2) different trials. $\mathrm{P} 1$ was severely paralyzed (cFMA $=25$ points; Fig. 2-A) and P2 was extreme severely paralyzed (cFMA=4 points; Fig. 2-B). The typical pattern of activity in each trial is depicted by a baseline period followed by an increase in 
WL amplitude indicating the movement onset. Afterwards the WL continues increasing until a maximum amplitude value (peak), indicating the dynamic contraction of the movement, and it is stabilized in a plateau of activity representing the postural maintenance. Subsequently the WL amplitude returns to baseline showing the finalization of the movement (Fig 1-B). WL amplitude during flexion of the upper arm resulted not only in higher EMG activity in forearm muscles than during finger extension (Fig. 2), but also more constant and stable across trials (Fig. 2-A).

We observed that in more than $25 \%$ of all trials during upper arm movement (across patients) the WL amplitude at forearm electrodes was higher than each forearm threshold (WL mean+std; i.e. muscle activity). Furthermore, we found that more of $10 \%$ of the patients presented WL amplitude bigger than each forearm threshold in more than $50 \%$ of the trials (Table 1).

Particularly, WL amplitude at the extensor carpi ulnaris during flexion of upper arm was higher than during finger extension in $60.2 \%$ of the trials across all patients (Table 1 ). Moreover, we also found that during flexion of the upper arm $64.4 \%$ of the patients showed a higher and more constant muscle contraction (in more than $50 \%$ of trials) of the extensor carpi ulnaris than during finger extension (Table 1).

\section{B. 95-percentile threshold in paretic upper limb.}

We found that during all upper arm movements the number of trials across patients in which the WL amplitude calculated at forearm electrodes exceeded the 95-percentile of WL (muscle activity) recorded at the same electrodes during forearm movements, was not bigger than $11 \%$. The percentage of patients that exceeded this threshold in more than $50 \%$ of the trials across forearm muscles was: a) in relation to finger extension: $5.93 \pm 1.28$ (flexion UA), $3.70 \pm 2.57$ (abduction), 6.67 \pm 2.22 (shoulder ext.), 2.22 \pm 0 (Supi/Pron); and b) in relation to wrist extension: $0.74 \pm 1.28$ (flexion UA), $0 \pm 0$ (abduction), 7.41 \pm 1.28 (shoulder ext.), $0 \pm 0$ (Supi/Pron) (Table 1.)

In particular, we found the maximum percentage of trials exceeding the 95-percentile threshold (calculated on EMG data acquired during fore arm movements) during elbow extension. The extensor carpi ulnaris and extensor digitorum during elbow extension presented WL amplitude values over the thresholds in $11.9 \%$ and $9.9 \%$ of trials respectively (Table $1)$.

\section{E. Control WL amplitude of non-paretic upper limb.}

For the non-paretic upper limb the forearm thresholds were calculated and compared against the mean WL amplitude of the non-paretic forearm muscles during upper arm movement. The percentage of patients that showed in more than $50 \%$ of the trials WL amplitude values higher during upper arm movements than the mean+std during forearm movements was $4.1 \%$ and than the 95 -percentile $0.65 \%$. These results indicate that the facilitation of forearm muscles activity during movements involving upper arm muscles is a unique characteristic of the paretic limb not present on the non-paretic one.

Independent sample t-test was used to calculate the statistical difference in the percentage of trials from the paretic and non-paretic upper limb where WL amplitude values of forearm electrodes were higher than each forearm threshold (mean \pm std and 95-percentile) during upper arm movements ( $p$-values are shown in Table. 1).
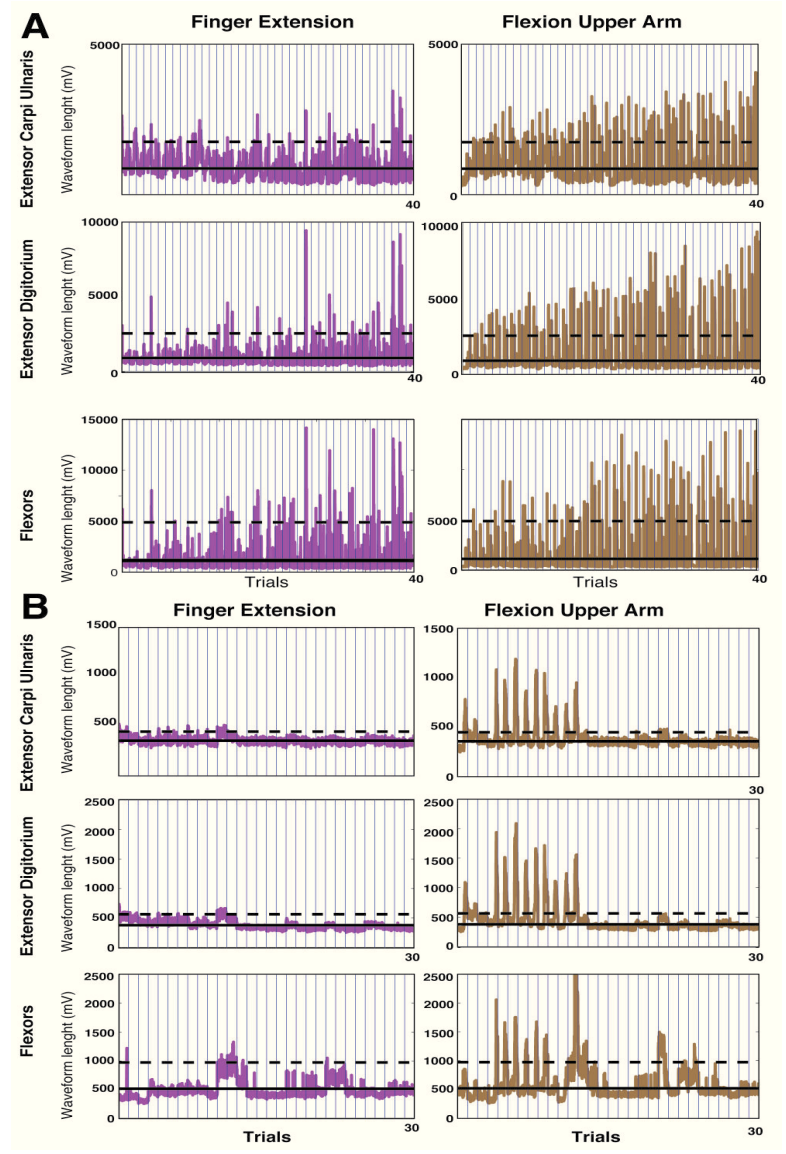

Figure 2. WL amplitude during: finger extension and upper arm flexion at three electrodes placed on the forearm muscles (from top to down): extensor carpi ulnaris, extensor digitorium and flexor digitorum. For each muscle the WL was calculated for each trial (delimited by the vertical blue lines). Two different stroke patients are shown: one severely impaired patient with cFMA scores of 25 points (A) and an extreme severely impaired patient with a cFMA scores of 4 points (B). For finger extension two different thresholds were calculated for each muscle across trials: the mean+std (continuous horizontal line) and the $95^{\text {th }}$ percentile (dashed horizontal line ), and applied to upper arm flexion movement in order to observe that the mean EMG amplitude during upper arm flexion was bigger and more stable than during finger extension.

In almost all comparisons the percentage of trials exceeding the respective threshold was significantly higher in the paretic limb than in the non-paretic one (mean p-value across forearm muscles and upper arm movements for the threshold mean \pm std: $0.01 \pm 0.051$, and for the 95 -percentile: $0.046 \pm 0.073)$. When using the threshold mean + std, we found that when comparing EMG activity between shoulder extension and wrist extension only, the percentage of trials exceeding this threshold was not significantly different between the paretic flexors and the non-paretic ones. When the 95-percentile was used as threshold, the difference between paretic and non-paretic limb became less significant $(0.046 \pm 0.073)$ than when using the mean + std threshold $(0.01 \pm 0.051)$ (Table 1). Furthermore, when using the 95percentile threshold there was no significant difference when 
comparing number of trials in which WL amplitude crossed the threshold (Table 1). However, there was a general tendency indicating that the percentage of trials exceeding the 95-percentile threshold were higher in the paretic limb than in the non-paretic one.

TABLE I. PERCENTAGE OF TRIALS AND PATIENTS EXCEEDING EACH THRESHOLD AND P-VALUE FROM THE COMPARISON BETWEEN PARETIC AND NON-PARETIC UPPER LIMB INFORMATION.

\begin{tabular}{|c|c|c|c|c|c|c|c|c|c|c|}
\hline \multirow{2}{*}{ MEASURE } & \multirow{2}{*}{ Thres } & \multirow{2}{*}{ Muscle } & \multicolumn{4}{|c|}{ Finger Extension } & \multicolumn{4}{|c|}{ Wrist Extension } \\
\hline & & & $\begin{array}{c}\text { Flex } \\
\text { UA } \\
\end{array}$ & $\begin{array}{c}\text { Abdu } \\
\text { UA }\end{array}$ & $\begin{array}{c}\text { Shoul } \\
\text { Ext. }\end{array}$ & Supi & $\begin{array}{l}\text { Flex } \\
\text { UAA }\end{array}$ & $\begin{array}{l}\text { Abd } \\
\text { uUA }\end{array}$ & $\begin{array}{l}\text { Shoul } \\
\text { Ext. }\end{array}$ & Supi \\
\hline \multirow{6}{*}{$\begin{array}{l}\text { PERCENTAGE } \\
\text { OF TRIALS } \\
\text { WHERE WL } \\
\text { AMPLITUDE } \\
\text { WAS LARGER } \\
\text { THAN EACH } \\
\text { FOREARM } \\
\text { THRESHOLD } \\
\text { DURING } \\
\text { UPPRR ARM } \\
\text { MOVEMENTS }\end{array}$} & \multirow{3}{*}{$\begin{array}{c}\text { Mean } \\
\text { +std }\end{array}$} & $\begin{array}{l}\text { Ext. } \\
\text { Ulnaris } \\
\end{array}$ & 60.2 & 52.4 & 53.4 & 39.5 & 47.7 & 37.5 & 37.2 & 29.2 \\
\hline & & $\begin{array}{l}\text { Ext. } \\
\text { Digit. }\end{array}$ & 50.6 & 39.9 & 52.5 & 36.7 & 39.9 & 29.2 & 36 & 28.9 \\
\hline & & Flexor & 46.9 & 37.9 & 44.3 & 32.9 & 40.1 & 26.6 & 37.2 & 25.5 \\
\hline & \multirow{3}{*}{$\begin{array}{c}95 \\
\text { \%tile }\end{array}$} & $\begin{array}{l}\text { Ext. } \\
\text { Ulnaris }\end{array}$ & 10.3 & 8.2 & 11.9 & 4.3 & 6.8 & 5.2 & 8.7 & 1.6 \\
\hline & & $\begin{array}{l}\text { Ext. } \\
\text { Digit. } \\
\end{array}$ & 6.4 & 3.9 & 9 & 3.3 & 4.3 & 2.9 & 9.8 & 3.2 \\
\hline & & Flexor & 8.9 & 3.6 & 8.6 & 4.1 & 3.9 & 5.2 & 6.9 & 1.3 \\
\hline \multirow{6}{*}{$\begin{array}{l}\text { PERCENTAGE } \\
\text { OF PATIENTS } \\
\text { THAT } \\
\text { PRESENTED IN } \\
\text { MORE THAN } \\
\text { 50\% OF THE } \\
\text { TRIALS A } \\
\text { LARGER WL } \\
\text { AMPLITUDE } \\
\text { THAN EACH } \\
\text { FOREARM } \\
\text { THRESHOLD } \\
\text { DURING } \\
\text { UPPER ARM } \\
\text { MOVEMENTS }\end{array}$} & \multirow{3}{*}{$\begin{array}{l}\text { Mean } \\
\text { +std }\end{array}$} & $\begin{array}{l}\text { Ext. } \\
\text { Ulnaris }\end{array}$ & 64.4 & 51.1 & 57.8 & 33.3 & 46.7 & 37.8 & 37.8 & 22.2 \\
\hline & & $\begin{array}{l}\text { Ext. } \\
\text { Digit. } \\
\end{array}$ & 44.4 & 37.8 & 53.3 & 26.7 & 40 & 24.4 & 31.1 & 22.2 \\
\hline & & Flexor & 44.4 & 40 & 40 & 17.8 & 37.8 & 26.7 & 24.4 & 8.9 \\
\hline & \multirow{3}{*}{$\begin{array}{c}95 \\
\% \text { tile }\end{array}$} & $\begin{array}{l}\text { Ext. } \\
\text { Ulnaris }\end{array}$ & 6.7 & 6.7 & 8.9 & 2.2 & 2.2 & 0 & 6.7 & 0 \\
\hline & & $\begin{array}{l}\text { Ext. } \\
\text { Digit. }\end{array}$ & 4.4 & 2.2 & 4.4 & 2.2 & 0 & 0 & 8.9 & 0 \\
\hline & & Flexor & 6.7 & 2.2 & 6.7 & 2.2 & 0 & 0 & 6.7 & 0 \\
\hline \multirow{6}{*}{$\begin{array}{l}\text { COMPARISON } \\
\text { BEETWEEN } \\
\text { THE NUMBER } \\
\text { OF TRIALS } \\
\text { PRESENTING } \\
\text { POSTURAL } \\
\text { EMG } \\
\text { FACILITATION } \\
\text { BETWEEN } \\
\text { PARETIC AND } \\
\text { NON-PARETIC } \\
\text { UPPER LIMB } \\
\text { (P-VALUE) }\end{array}$} & \multirow{3}{*}{$\begin{array}{c}\text { Mean } \\
+ \text { +std }\end{array}$} & $\begin{array}{l}\text { Ext. } \\
\text { Ulnaris }\end{array}$ & $\begin{array}{l}6.83 \\
\mathrm{e}-09\end{array}$ & $\begin{array}{l}4.92 \\
\mathrm{e}-07\end{array}$ & $\begin{array}{r}9.59 \\
\mathrm{e}-11\end{array}$ & $\begin{array}{l}3.89 \\
\mathrm{e}-07\end{array}$ & $\begin{array}{l}1.20 \\
\mathrm{e}-12\end{array}$ & $\begin{array}{l}3.04 \\
e-10\end{array}$ & $\begin{array}{l}1.83 \\
\mathrm{e}-09\end{array}$ & $\begin{array}{l}4.20 \\
\text { e-12 }\end{array}$ \\
\hline & & $\begin{array}{l}\text { Ext. } \\
\text { Digit. }\end{array}$ & $\begin{array}{l}1.32 \\
\mathrm{e}-10 \\
\end{array}$ & $\begin{array}{r}1.29 \\
\mathrm{e}-08 \\
\end{array}$ & $\begin{array}{r}8.68 \\
\text { e-14 } \\
\end{array}$ & $\begin{array}{r}9.59 \\
\mathrm{e}-13 \\
\end{array}$ & $\begin{array}{r}6.02 \\
\text { e-12 } \\
\end{array}$ & $\begin{array}{r}2.31 \\
\mathrm{e}-10 \\
\end{array}$ & $\begin{array}{r}2.51 \\
\mathrm{e}-09 \\
\end{array}$ & $\begin{array}{l}1.81 \\
\mathrm{e}-11 \\
\end{array}$ \\
\hline & & Flexor & $\begin{array}{r}1.40 \\
\text { e-10 } \\
\end{array}$ & $\begin{array}{r}2.50 \\
\text { e-12 } \\
\end{array}$ & $\begin{array}{r}8.20 \\
e-07 \\
\end{array}$ & $\begin{array}{r}2.83 \\
\mathrm{e}-10\end{array}$ & $\begin{array}{l}1.80 \\
e-07\end{array}$ & $\begin{array}{l}2.23 \\
\mathrm{e}-07\end{array}$ & 0.250 & $\begin{array}{l}1.16 \\
e-07\end{array}$ \\
\hline & \multirow{3}{*}{$\begin{array}{c}95 \\
\text { \%tile }\end{array}$} & $\begin{array}{l}\text { Ext. } \\
\text { Ulnaris }\end{array}$ & $\begin{array}{c}1 \\
e-03\end{array}$ & 0.154 & 0.041 & 0.09 & $\begin{array}{c}1 \\
e-03\end{array}$ & $\stackrel{2}{2}$ & 0.046 & 0.08 \\
\hline & & $\begin{array}{l}\text { Ext. } \\
\text { Digit. }\end{array}$ & $\begin{array}{c}8 \\
e-03\end{array}$ & 0.011 & 0.054 & 0.03 & 0.01 & 0.02 & 0.034 & 0.03 \\
\hline & & Flexor & $\begin{array}{c}3 \\
e-03\end{array}$ & 0.033 & 0.050 & 0.03 & $\begin{array}{c}2 \\
\mathrm{e}-03\end{array}$ & $\stackrel{2}{\stackrel{2}{e-03}}$ & 0.348 & 0.02 \\
\hline
\end{tabular}

Thresh: Threshold, Mean+std: mean+standard deviation, 95 \%tile: 95 -percentile, Ext. Ulnaris: Extensor carpi ulnaris, Ext. Digit.: Extensor Digitorium; Flexor: Flexor Digitorum, Flex UA: flexion upper arm, Abduct. UA: abdu upper arm, Shoul Ext.: shoulder extension, Supi: supination/pronation. $\mathrm{P}$-values in bold are statistically significant, with dark gray $\mathrm{p} \leq 0.001$, middle gray $\mathrm{p} \leq 0.01$ and light dotted gray $\mathrm{p} \leq 0.05$, e means exponent base 10 .

\section{CONCLUSION}

We have demonstrated that severely affected chronic stroke patients without remaining finger extension can facilitate the control and strength of forearm muscles contraction by simultaneously activating upper arm muscles (involving less paralyzed muscles). This effect was only present in the paretic upper limb. Our results suggest that remaining connections between the brain and completely paralyzed distal muscles (more compromised) exist. However, the control and activation of these connections is affected by erratic muscle synergies produced by the brain lesion. Our findings are in agreement with previous work [6], [7] indicating that the positive influence of specific recruitment of less paralyzed muscles of the upper limb through (specific upper limb postures) in forearm muscles activation. Coordinated simultaneous movements involving distal (upper arm) and proximal (forearm) muscle contractions should be considered as a potential strategy in stroke motor rehabilitation to restore control of more affected muscles. Furthermore, some standard clinical scales should consider arm posture since the increase in EMG amplitude could result in visible movement. This means, the transition from completely paralyzed to some movement could depend on more proximal muscles contraction level. With more controllable muscle activity patients could profit better from rehabilitation strategies based on EMG biofeedback. Feedback contingency will reinforce residual muscle activity and might allow long-term effects and generalization to untrained tasks.

\section{AKNOWLEDGMENTS}

The authors thank Woosang Cho, Ozge Yilmaz, Fabricio Lima Brasil, Marco Curado, Manuel Agostini, for their assistance during EMG data recording, and Doris Broetz for the FMA scale assessment.

\section{REFERENCES}

[1] G. Gresham, M. Kelly-Hayes, P. Wolf, A. Beiser, C. Kase, and R. D'Agostino, "Survival and functional status of 20 or more years after first stroke: The Framingham study. Stroke," Stroke, vol. 29, pp. 793-797, 1995.

[2] P. Langhorne, F. Coupar, and A. Pollock, "Motor recovery after stroke: a systematic review.," Lancet neurology, vol. 8, no. 8, pp. 741-54, Aug. 2009.

[3] P. Kung, C. K. Lin, M. Ju, and S. Chen, "Reducing Abnormal Synergies of Forearm, Elbow, and Shoulder Joints in Stroke Patients with Neuro-rehabilitation Robot Treatment and Assessment," Journal of Medicl and Biological Engineering, vol. 32, no. 2, pp. 139-146, 2009.

[4] S. Brunnstrom, Movement therapy in hemiplegia. New York: Harper \& Row, 1970

[5] L. Dipietro, H. I. Krebs, S. E. Fasoli, B. T. Volpe, J. Stein, C Bever, and N. Hogan, "Changing motor synergies in chronic stroke." Journal of neurophysiology, vol. 98, no. 2, pp. 757-68, Aug. 2007.

[6] S. Bobath, Adult hemiplegia: evaluation and treatment. London: Willian Heinemann Medical Books, 1970.

[7] A. Aruin, "The effect of asymmetry of posture on anticipatory postural adjustments," Neuroscience Letters, vol. 401, pp. 150$153,2006$.

[8] R. F. Beer, J. P. Dewald, and W. Z. Rymer, "Deficits in the coordination of multijoint arm movements in patients with hemiparesis: evidence for disturbed control of limb dynamics.," Experimental brain research., vol. 131, no. 3, pp. 305-19, Apr. 2000.

[9] J. V. Graham, C. Eustace, K. Brock, E. Swain, and S. IrwinCarruthers, "The Bobath concept in contemporary clinical practice," Topics in stroke rehabilitation, vol. 16, no. 1, pp. 5768, 2009.

[10] A. Ramos-Murguialday, D. Broetz, M. Rea, L. Läer, O. Yilmaz, F. L. Brasil, G. Liberati, M. R. Curado, E. Garcia-Cossio, A. Vyziotis, W. Cho, M. Agostini, E. Soares, S. Soekadar, A. Caria, L. G. Cohen, and N. Birbaumer, "Brain-machine-interface in chronic stroke rehabilitation: A controlled study.," Annals of neurology, Mar. 2013.

[11] K. A. Farry, I. D. Walker, and R. G. Baraniuk, "Myoelecric Teleoperarion of a complex robotic hand," IEEE Transactions of Robotics and Automation, vol. 12, no. 5, pp. 775-787, 1996. 